\title{
ESTIMACIÓN DE RIESGO DE EXPOSICIÓN A METALES PESADOS POR CONSUMO DE PLECOS (Pterygoplichthys spp.) EN INFANTES DE COMUNIDADES RIBEREÑAS DE LOS RÍOS GRIJALVA Y USUMACINTA, MÉXICO
}

\author{
Habacuc LORENZO-MÁRQUEZ ${ }^{1}$, Arturo TORRES-DOSAL ${ }^{1 *}$, Everardo BARBA MACÍAS ${ }^{2}$, \\ César Arturo ILIZALITURRI HERNÁNDEZ ${ }^{3}$, Rebeca Isabel MARTÍNEZ-SALINAS ${ }^{4}$, \\ Juan Jesús MORALES LÓPEZ ${ }^{1}$ y Irene SÁNCHEZ MORENO ${ }^{5}$
}

${ }^{1}$ El Colegio de la Frontera Sur, Unidad San Cristóbal de Las Casas, Carretera Panamericana y Periférico Sur s/n, Barrio María Auxiliadora, San Cristóbal de las Casas, Chiapas, México, C.P. 29290

${ }^{2}$ El Colegio de la Frontera Sur, Unidad Villahermosa, Carretera Villahermosa-Reforma Km 15.5, Ranchería Guineo, Sección II, Villahermosa, Tabasco, México, C.P. 86280

${ }^{3}$ Centro de Investigación y Aplicación en Ambiente y Salud, Universidad Autónoma de San Luis Potosí. Edificio de Laboratorio Nacional, Unidad de Posgrado, Avenida Sierra Leona \# 550, Colonia Lomas 2a. Sección, San Luis Potosí, San Luis Potosí, México, C.P. 78210

${ }^{4}$ Escuela de Ingeniería Ambiental, Universidad de Ciencias y Artes de Chiapas, Libramiento Norte Poniente \# 1150, Colonia Lajas Maciel, Tuxtla Gutiérrez, Chiapas, México, C.P. 29000

${ }^{5}$ Facultad de Ciencias de la Nutrición y Alimentos, Universidad de Ciencias y Artes de Chiapas, Libramiento Norte Poniente \# 1150, Colonia Lajas Maciel, Tuxtla Gutiérrez, Chiapas, México, C.P. 29000

*Autor para correspondencia: atorres@ecosur.mx

(Recibido marzo 2015; aceptado noviembre 2015)

Palabras clave: cuenca Grijalva-Usumacinta, pez invasor, Monte Carlo, IEUBK

\section{RESUMEN}

El objetivo de este estudio fue cuantificar la concentración de metales pesados $(\mathrm{Cd}, \mathrm{Cr}$, $\mathrm{Mn}$ y $\mathrm{Pb}$ ) en tejido muscular del pez invasor Pterygoplichthys spp. y estimar el riesgo por exposición oral a estos elementos en infantes usando modelación probabilística de escenarios por el método Monte Carlo en las subcuencas Grijalva y Usumacinta en los estados de Chiapas y Tabasco, México. Se obtuvieron las siguientes concentraciones promedio de metales en tejido muscular en $\mu \mathrm{g} / \mathrm{kg}: \mathrm{Mn}=734.70, \mathrm{Cr}=315.16, \mathrm{~Pb}=$ 204.62 y Cd $=38.04$. Las dosis de exposición estimadas mostraron cocientes de riesgo bajos para ambas subcuencas. Se utilizó el modelo biocinético de exposición integral al plomo (IEUBK, por sus siglas en inglés) para estimar la concentración sanguínea de $\mathrm{Pb}$ en infantes de 36 a 60 meses de edad, se calculó el porcentaje de población con niveles $>5 \mu \mathrm{g} \mathrm{Pb} / \mathrm{dL}$ en sangre el cual fue de: 2.792 y 7.254 para las subcuencas Grijalva y Usumacinta respectivamente. Ambos resultados muestran que existe un riesgo de exposición a plomo en infantes. Sin embargo, es necesario tener un estudio más detallado en el que se consideren factores como la edad y la talla de los peces capturados, así como evaluar la concentración en subproductos como la harina de pescado, la influencia en la concentración de los procesos de cocinado y la asociación con la exposición a xenobióticos.

Key words: Grijalva-Usumacinta basin, invader fish, Monte Carlo, IEUBK 


\begin{abstract}
The aim of this study was to assess the concentration levels in muscle tissue of invasive fish Pterygoplichthys spp. and to estimate the risk of exposure to heavy metals $(\mathrm{Cd}, \mathrm{Cr}, \mathrm{Mn}$ and $\mathrm{Pb})$ in infants by ingestion using probabilistic scenarios modeling in the sub-basins Grijalva and Usumacinta located in the states of Chiapas and Tabasco, Mexico. The following average metal concentrations in muscle tissue were obtained in $\mu \mathrm{g} / \mathrm{kg}: \mathrm{Mn}=734.70, \mathrm{Cr}=315.16=204.62 \mathrm{~Pb}$ and $\mathrm{Cd}=38.04$. Exposure dose to $\mathrm{Cd}, \mathrm{Cr}$ and $\mathrm{Mn}$ were estimated using Monte Carlo analysis. The analysis showed low risk quotient for both sub-basins. Because there has not been reported reference dose of exposure to $\mathrm{Pb}$ we used the Integrated Exposure Uptake Biokinetic Model (IEUBK) to estimate infant (36-60 months old) blood concentrations of $\mathrm{Pb}$ by eating fish. Results are shown as percentage of population $>5 \mu \mathrm{g} \mathrm{Pb} / \mathrm{dL}$ of blood 2.792 and 7.254 for subbasins Grijalva and Usumacinta respectively. Both values indicate that there is a risk in children. However, it is necessary to have a detailed study that considers factors such as age and size of the fish taken, as well as evaluate the concentration in sub-products such as fishmeal and influence in the concentration of the cooking processes.
\end{abstract}

\section{INTRODUCCIÓN}

Actualmente se considera que la invasión biológica es uno de los causantes más importantes de la destrucción de hábitats y la fragmentación del paisaje e incluso está considerada como un vector más del cambio global (Dukes y Mooney 1999, Mooney y Hobbs 2000), aunque su impacto sobre los entornos y la sociedad en general es aún mucho menor que otros fenómenos como la alteración de la composición atmosférica, la contaminación o la pérdida de suelo (Castro et al. 2004). La invasión biológica se refiere al proceso de introducción, establecimiento y expansión de especies exóticas procedentes de otras áreas geográficas. Uno de los mecanismos comúnmente aceptados de invasión por especies exóticas es la propuesta en la hipótesis del escape de los enemigos naturales (EEN) por Keane y Crawley (2001), en la cual se plantea que las especies invasoras experimentan tras su introducción en una región fuera de su ambiente natural una disminución de la regulación que sobre ellas ejercían sus enemigos naturales (depredadores, parásitos o patógenos). Esto propicia que la especie invasora aumente su población y su rango de distribución rápidamente (Santamaría et al. 2008).

En México, desde hace 20 años se ha desarrollado un problema de invasión biológica provocado por el género Pterygoplichthys conocido comúnmente como "pleco", "plecos" o "pez diablo". Pterygoplichthys es endémico de Sudamérica y pertenece a la familia Loricariidae (Mendoza et al. 2007). Esta familia es de las más diversas, con 716 especies descritas (Nelson 2006, Ferraris 2007), y debido a sus cualidades detritívoras algunas especies han sido comercializadas como peces ornamentales y controladores de algas (Hossain et al. 2008). Lo anterior ha promovido su introducción en ríos y lagos de regiones de clima cálido, ya sea de forma controlada o accidental (Hoover et al. 2004, Nico et al. 2009). Además, gracias a diversas adaptaciones biológicas, como una alta fertilidad, una reproducción precoz así como tolerancia a aguas con condiciones variantes de calidad, tiene ventajas frente a las especies nativas (Nico y Martín 2001, Chávez et al. 2006) por lo cual representa un riesgo biológico en donde se encuentre. En México se reportó el primer avistamiento en el año 1995 en el río Mezcala en el estado de Guerrero (Guzmán y Barragán 1997). Actualmente Pterygoplichthys spp. se encuentra distribuido en distintos puntos de México como en la presa El Infiernillo y el río Balsas en el estado de Michoacán (Contreras et al. 2006). Resaltan los casos específicos reportados en el sureste del país: la cuenca alta del Grijalva en Tecpatán, Chiapas (Mendoza et al. 2007, Ramírez-Guevara y Rodiles-Hernández 2013), la Laguna de las Ilusiones en Villahermosa, Tabasco (Wakida-Kusunoki et al. 2007), los ríos de la Sierra y los Pantanos de Centla, en el estado de Tabasco (Barba et al. 2014), la Laguna de Catazajá, Chiapas y los humedades de la Libertad, Palenque en la misma entidad (Ramírez-Guevara y Rodiles-Hernández 2013), las Lagunas el Susil, Suñiná y el río San Pedro, en Balancán, Tabasco (Cano et al. 2012), así como el río Palizada en Campeche (Wakida-Kusunoki y Amador-del Ángel 2011).

La amplia distribución que ha alcanzado este género comenzó a causar problemas ambientales como la degradación del hábitat, hibridación, deterioro de la calidad del agua, e introducción de parásitos 
(Collares-Pereira et al. 2002). Así como serias alteraciones para la pesca en las comunidades ribereñas debido a que especies de interés comercial son desplazadas y las redes utilizadas por los pescadores son destruidas (Mendoza et al. 2007).

En respuesta a estos problemas, algunas investigaciones que abordan el tema del plecos se han orientado a su aprovechamiento como alimento para las poblaciones en general, o bien, para obtener subproductos como fertilizantes, ensilado de pescado como complemento alimenticio para ganado, así como harina de pescado para alimento de peces. La opción más promovida entre las comunidades pesqueras para su aprovechamiento es la del consumo del pez como parte de la dieta, aunque sin mucha aceptación por el momento (Paramo et al. 2008, Cano et al. 2012, Escalera-Gallardo et al. 2012).

Por otra parte, los ambientes acuáticos son altamente vulnerables a contaminantes debido a las actividades antrópicas derivadas del incremento de diferentes actividades humanas. siendo los ríos principales vertederos de los desechos industriales, domésticos o agrícolas. Los metales pesados son ampliamente utilizados en la industria y en los últimos años han aumentado los problemas por la exposición a estos elementos. En México, la inadecuada planeación y el escaso control sobre el uso de los recursos naturales, así como la rápida industrialización, el crecimiento desordenado de sus principales ciudades $\mathrm{y}$ en algunos casos los abundantes aportes naturales, principalmente del intemperismo geológico, han sido los factores responsables del aumento de los niveles de concentración de metales en cuerpos de agua (Villanueva y Botello 1998, Flores y Albert 2004, Frías et al. 2010).

La cuenca Grijalva-Usumacinta no es la excepción, puesto que a lo largo de ella existen grandes asentamientos humanos y se desarrollan diversas actividades económicas como la minería en pequeña escala y la agricultura, lo que expone a los organismos acuáticos a diversos contaminantes, fundamentalmente metales pesados. Estos últimos son de particular preocupación debido a su capacidad para formar complejos con la materia orgánica y por su tendencia a fijarse en los tejidos de los organismos expuestos. Este proceso conocido como bioacumulación ocurre a través de diferentes vías como la ingestión sistemática de partículas de sedimento y a través de la cadena alimentaria. Lo anterior se considera uno de los problemas más graves que pueden generar dichos elementos en los medios acuáticos (Newarman 1998, Villanueva y Botello 1998, Bervotes et al. 2001, Burger et al. 2002).
Algunos organismos de hábitos bentónicos y filtradores, debido a sus altas tasas de absorción, son capaces de retener en su cuerpo concentraciones de metales proporcionalmente mayores a las del medio que les rodea (Dhanakumar et al. 2015). El peligro de aquellos elementos metálicos tóxicos es derivado de su ingreso en las cadenas tróficas, donde se vuelve un potencial problema de salud humana al ingerir organismos con alta carga corporal de contaminantes. La alta exposición a xenobióticos de manera frecuente puede representar un peligro para los consumidores. Los infantes son los más vulnerables debido a que sus sistemas corporales aún se están desarrollando, presentan cambios rápidos en el crecimiento, tienen variaciones en la madurez orgánica y tisular así como un déficit cuantitativo y cualitativo en su sistema de inmunovigilancia. Además, están más expuestos que los adultos porque proporcionalmente consumen más alimento por kilogramo de peso, beben más líquidos y respiran más aire (Zayas y Cabrera 2007, Frías et al. 2010).

El pleco al tener hábitos detritívoros está particularmente expuesto a la ingesta de metales pesados asociados a las partículas de sedimento de las cuales se alimenta. Esta condición y el hecho de que es una especie propuesta para incluirse en la dieta regular, permite establecer la importancia de realizar un estudio que valore la potencial exposición a metales pesados por esta ruta en individuos vulnerables como los infantes.

El objetivo del presente estudio fue estimar el riesgo de exposición a cadmio $(\mathrm{Cd})$, cromo $(\mathrm{Cr})$, manganeso $(\mathrm{Mn})$ y plomo $(\mathrm{Pb})$ por ingesta de Pterygoplichthys spp. en la cuenca baja del GrijalvaUsumacinta. Se evaluó el contenido metálico en tejido muscular de Pterygoplichthys spp. en diferentes sitios de las subcuencas Grijalva y Usumacinta y se estimaron los posibles riesgos para la salud en niños de 36 a 60 meses de edad a través de la metodología de simulación probabilística Monte Carlo y del modelo biocinético de exposición integral al plomo (IEUBK, por sus siglas en inglés).

\section{MATERIALES Y MÉTODOS}

\section{Área de estudio}

Los ríos Grijalva y Usumacinta son parte de la cuenca hidrológica transfronteriza Grijalva-Usumacinta localizada en la parte sureste de México y noreste de Guatemala. Son dos de los ríos más caudalosos de México con un escurrimiento promedio de 115536 millones de $\mathrm{m}^{3} /$ año y son de gran importancia 
debido a que dotan de recursos hídricos a varias ciudades, además son de los ríos con mayor longitud en México con $1521 \mathrm{~km}$ y un área total de $83553 \mathrm{~km}^{2}$. El estudio se llevó a cabo en los estados de Chiapas y Tabasco, México, en sitios donde se ha reportado la presencia de Pterygoplichthys spp., dentro de las dos subcuencas. Los puntos seleccionados fueron los siguientes: 15Q 662123.88 E 1936581.00 N Tenosique, Tabasco (UT); 15Q 655184.09 E $1969187.35 \mathrm{~N} \mathrm{Ba}-$ lancán, Tabasco (BA); 15Q 604662.31 E 1960279.24 N Catazajá, Chiapas (CC); 15Q 540921.00 E 2033390.00 N Centla, Tabasco (CE); 15Q 441319.30 E 1918158.68 N Tecpatán, Chiapas (EH); 15Q 493179.38 E 1978399.54 N Villahermosa, Tabasco (VH); 15Q 499285.00 E 1955005.00 N Teapa, Tabasco (LSG); 15Q 433502.72 E 2014193.84 N Cárdenas, Tabasco (ZP) y 15Q 462712.11 E $1983620.32 \mathrm{~N}$ Cárdenas, Tabasco (CT), en sitios donde hay comunidades con actividades mayoritariamente dedicadas a la pesca y en las que su dieta principal la constituye el consumo de pescado (Fig. 1).

\section{Muestreo}

El muestreo fue realizado entre la primavera y el verano de 2014 y se llevó a cabo con ayuda de los pescadores locales. Se colectaron un total de 240 ejemplares de Pterygoplichthys spp. Adultos, los que fueron sacrificados para obtener el tejido muscular. El tejido muscular obtenido se colocó en frascos ámbar con taparrosca, se mantuvieron a $4^{\circ} \mathrm{C}$ para su transporte y posterior congelación a $-4{ }^{\circ} \mathrm{C}$ hasta su análisis.

\section{Digestión ácida}

La extracción de metales se llevó a cabo por digestión ácida asistida por microondas (Anton Paar Multiwave 3000) según las especificaciones del método USEPA 3052 (USEPA 1996). Se pesaron $2 \mathrm{~g}$ de tejido muscular de Pterygoplichthys spp., se añadieron $3 \mathrm{~mL}$ de $\mathrm{HClO}_{4}$ y $9 \mathrm{~mL}$ de $\mathrm{HNO}_{3}$ marca JT Baker grado instra para el análisis de metales traza. Las muestras se enfriaron hasta temperatura ambiente y se aforaron a $25 \mathrm{~mL}$ con $\mathrm{HNO}_{3} 0.2 \%$.

\section{Cuantificación de metales traza}

La cuantificación de metales traza se realizó de acuerdo con el método USEPA 7010 (USEPA 2007a) por medio de espectrometría de absorción atómica con horno de grafito (modelo Varian SpectrAA 220 y Varian GTA 110) con los siguientes límites de detección $\mathrm{Cd}=0.2 \mu \mathrm{g} / \mathrm{L}, \mathrm{Cr}=2 \mu \mathrm{g} / \mathrm{L}, \mathrm{Mn}=5 \mu \mathrm{g} / \mathrm{L}$ y $\mathrm{Pb}=10 \mu \mathrm{g} / \mathrm{L}$. Como control de calidad se utilizó el material de referencia del Instituto Nacional de

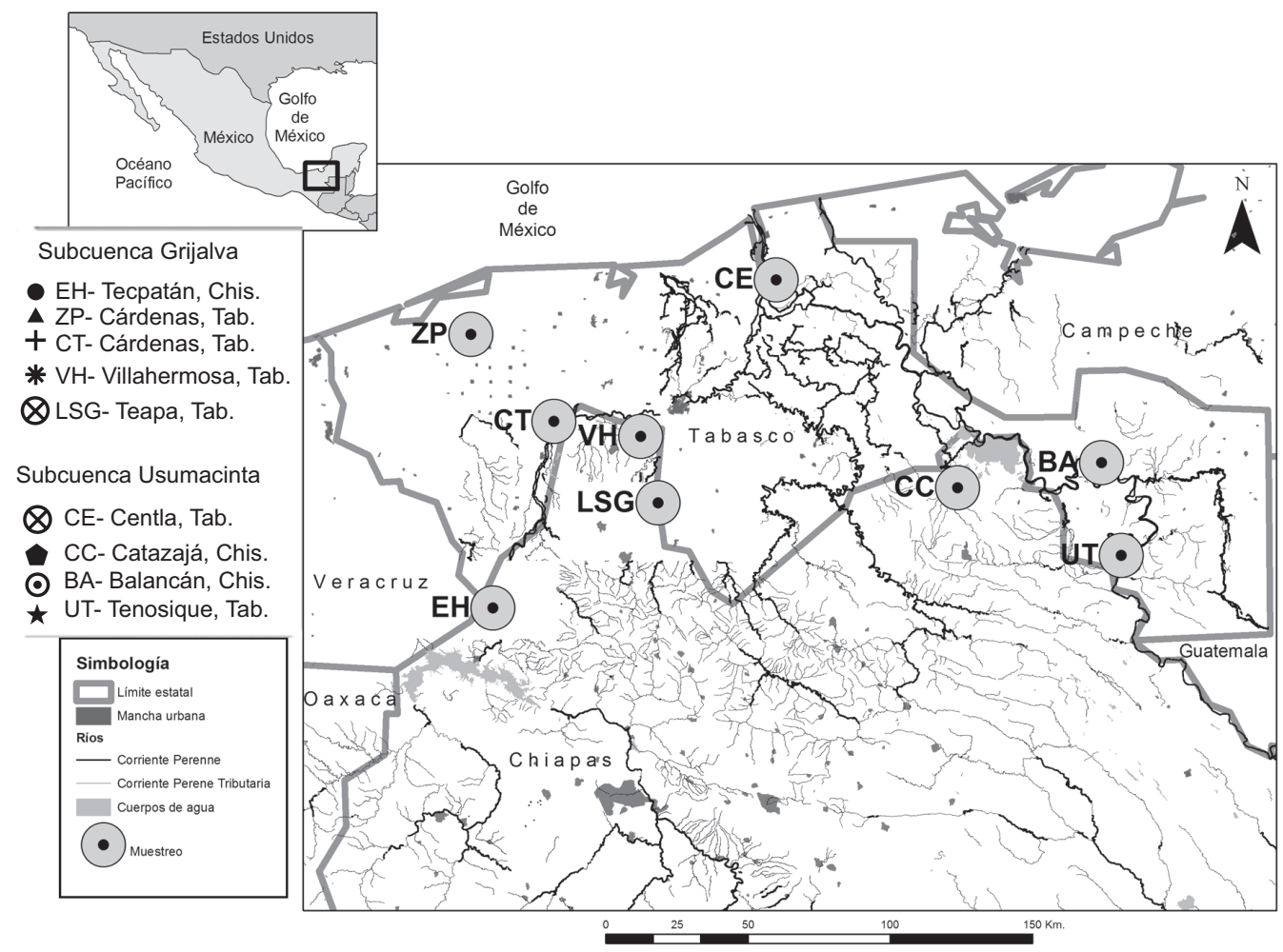

Fig. 1. Localización de los puntos de muestreo en las subcuencas Grijalva y Usumacinta 
Estándares y Tecnología (NIST, por sus siglas en inglés) de tejido de hígado bovino $1577 \mathrm{c}$; se obtuvieron porcentajes de recuperación de 95 a $97 \%$.

\section{Análisis estadístico}

Se realizó la prueba de Kolmogorov-Smirnov para verificar la condición de normalidad de las distribuciones de los datos y la prueba de Levene para evaluar la homogeneidad de varianza. Se realizaron transformaciones logarítmicas para corregir la heterogeneidad en los casos necesarios. Se realizó un análisis de varianza de una vía para cada uno de los metales cuantificados con la finalidad de determinar la existencia de diferencias significativas $(\mathrm{p}<0.05)$ entre los sitios muestreados. El análisis se realizó con el paquete JMP Statistical Discovery ${ }^{\mathrm{TM}}$, SAS versión 9.

\section{Estimación de riesgo por ingesta, método determinístico}

Para la estimación de riesgo por ingesta se utilizó como guía el método determinístico descrito en la Metodología de Identificación y Evaluación de Riesgos para la Salud en Sitios Contaminados, de la Organización Panamericana de la Salud (DíazBarriga 1999) en la cual es necesario obtener una dosis de ingesta del contaminante considerando algunos aspectos como los que se describen en la siguiente ecuación:

Dosis $=\frac{\text { Conc. } * T I}{P C} * F E$

\section{Dónde:}

Dosis $=$ Dosis estimada de exposición (mg/kg-día). Conc.$=$ Concentración ambiental del contaminante en el medio analizado $(\mathrm{mg} / \mathrm{kg})$.
$T I=$ Tasa de ingesta diaria del elemento del medio contaminado, en este caso tejido muscular de Pterygoplichthys spp. ( $\mathrm{mg} / \mathrm{kg})$

$P C=$ Peso corporal de la población receptora $(\mathrm{kg})$. $F E=$ Factor de exposición $=1$, máximo riesgo.

Los datos de TI y PC fueron consultados en el Manual de Factores de Exposición Específicos para Niños (USEPA 2008; Cuadro I) utilizando los datos para infantes de 36 a 60 meses de edad en ambos casos.

El siguiente paso fue dividir cada dosis estimada de exposición entre la dosis de referencia (RfD) para obtener un cociente de riesgo (CR), como podemos ver a continuación:

$\mathrm{CR}=\frac{\text { Dosis de exposición }}{\mathrm{RfD}}$

La interpretación del nivel de riesgo se basa en que un $\mathrm{CR}>1$ significa que la exposición estimada para un individuo supera los límites de seguridad propuestos para el contaminante de interés (RfD), por lo que hay un riesgo alto de manifestar los efectos adversos descritos para cada contaminante. Mientras que un $\mathrm{CR}<1$ significa que el riesgo de exposición es bajo por lo que la posibilidad de ocurrencia de un efecto adverso en la población es mínima.

\section{Estimación de riesgo por ingesta, método probabilístico}

En la estimación de riesgo, hay varias fuentes de variabilidad. Debido a la diversificación y variación inherente de los parámetros de la ecuación que explica el proceso de riesgo, estas variables se pueden definir en términos de una función de densidad de probabilidad derivada de un número limitado de

CUADRO I. PARÁMETROS UTILIZADOS PARA ESTIMAR LA EXPOSICIÓN POR INGESTA DE Cd, Cr Y Mn EN INFANTES EN LAS SUBCUENCAS GRIJALVA-USUMACINTA

\begin{tabular}{|c|c|c|c|c|c|c|c|c|c|c|}
\hline Parámetros & Comentarios & Distribución & $n$ & Mínimo & Máximo & Media & $\mathrm{DE}$ & Mediana & $P_{25}$ & $P_{95}$ \\
\hline $\mathrm{Cd}$ en peces $(\mathrm{mg} / \mathrm{kg})$ & Muestreo & Log Normal & 240 & - & - & 0.038 & 0.008 & 0.03 & 0.02 & 0.12 \\
\hline $\mathrm{Cr}$ en peces $(\mathrm{mg} / \mathrm{kg})$ & Muestreo & Log Normal & 240 & - & - & 0.315 & 0.192 & 0.20 & 0.12 & 0.72 \\
\hline Mn en peces $(\mathrm{mg} / \mathrm{kg})$ & Muestreo & Log Normal & 240 & - & - & 0.734 & 0.984 & 0.17 & 0.07 & 1.54 \\
\hline $\mathrm{Pb}$ en peces $(\mathrm{mg} / \mathrm{kg})$ & Muestreo & - & 240 & - & - & 0.204 & 0.141 & 0.15 & 0.07 & 0.453 \\
\hline TI de peces (kg/día) & USEPA (2008) & Triangular & - & 0.041 & 0.048 & 0.045 & - & - & - & - \\
\hline PC niños (kg) & USEPA (2008) & Triangular & - & 13.5 & 26.2 & 18.6 & - & - & - & - \\
\hline RfD Cd oral (mg/kg/día) & \multicolumn{5}{|c|}{ Proteinuria significativa USEPA (1994b) } & 0.0005 & - & - & - & - \\
\hline RfD Cr oral (mg/kg/día) & \multicolumn{5}{|c|}{ Necrosis celular USEPA (1998b) } & 1.5 & - & - & - & - \\
\hline RfD Mn oral (mg/kg/día) & \multicolumn{5}{|c|}{ Efectos en el sistema nervioso central USEPA (1996b) } & 0.14 & - & - & - & - \\
\hline
\end{tabular}

$\mathrm{TI}=$ Tasa de ingesta, $\mathrm{PC}=$ Peso corporal, $\mathrm{RfD}=$ Dosis de referencia 
observaciones. Este recurso permite que a partir de un número reducido de muestras se pueda modelar un fenómeno completo con gran certeza debido a la generación de números aleatorios para cada parámetro específico, con el que se puede explicar matemáticamente el proceso o fenómeno total (USEPA 1997).

La mayor incertidumbre de este abordaje deriva de la no cuantificación del tóxico directamente en los individuos de interés (en este caso niños). Sin embargo, es una aproximación preliminar aceptable para la toma de decisiones en la prevencion de riesgos para la salud.

De este modo, para tener en cuenta de forma explícita la variabilidad de los parámetros de la ecuación en la estimación del cociente de riesgo, se adoptó una simulación de Monte Carlo mediante el programa Oracle Crystal Ball (Versión 11.1.2.3.500 de Microsoft Office) para analizar los datos. Se utilizaron diez mil iteraciones para asegurar la estabilidad de los resultados.

\section{Modelo biocinético de exposición integral al plomo (IEUBK)}

Debido a que no se ha reportado un umbral de toxicidad para $\mathrm{Pb}$, existen otros métodos por los cuales se puede calcular el porcentaje de población asociada a niveles de exposición específicos. Para esto, la Agencia de Protección al Ambiente de EUA (USEPA, por sus siglas en inglés) propone la utilización del modelo biocinético de exposición integral al plomo conocido como IEUBK, por sus siglas en inglés, el cual permite estimar una posible distribución de la concentración de plomo en sangre para un niño o una población de niños expuestos. El modelo requiere información sobre la exposición al plomo e información sobre el niño o la población de niños que desea estudiarse. Para ello, el IEUBK utiliza valores y ecuaciones que consideran la exposición, la absorción y la biocinética del plomo en la población infantil. El IEUBK puede ser de utilidad para inferir estrategias de restauración en sitios contaminados con plomo. Sin embargo, se requiere cautela en su uso, ya que el modelo por sí sólo no sirve para definir los niveles de seguridad ambiental pues siempre será requerida la validación en escenarios reales (USEPA 1994a).

El modelo fue alimentado sólo con los datos disponibles del sitio, procurando ser muy conservadores con las consideraciones de los parámetros del modelo (USEPA 2007b). De este modo se consideró: aire "condición base" (concentración en aire $0.1 \mu \mathrm{g} / \mathrm{m}^{3}$, tiempo de permanencia al aire libre, 4 horas); agua "condición base" (concentración en agua, $4 \mu \mathrm{g} / \mathrm{L}$, consumo diario de agua, $0.59 \mathrm{~L} /$ día); concentración de plomo en sangre de madres en el parto "condición base" (1 $\mu \mathrm{g} \mathrm{Pb} / \mathrm{dL})$; suelo/polvo (concentración en suelo de $15.375 \mu \mathrm{g} / \mathrm{g}$ para los sitios correspondientes al río Grijalva y $15.736 \mu \mathrm{g} / \mathrm{g}$ para los sitios correspondientes al río Usumacinta; SGM 2015); dieta (se utilizaron los valores promedio para cada metal en ambas subcuencas con un porcentaje de consumo de pescado en la dieta del $100 \%$, asumiendo que ésta es su única fuente proteica). Los análisis se ajustaron a niños de 36 a 60 meses de edad. Se utilizaron 5 y $10 \mu \mathrm{g} \mathrm{Pb} / \mathrm{dL}$ de sangre como umbrales de exposición a los que se han descrito efectos adversos en el sistema nervioso central y disminución de la inteligencia y del desarrollo neuroconductual, respectivamente (USEPA 1998a).

\section{RESULTADOS Y DISCUSIÓN}

\section{Concentración de metales pesados en tejido mus- cular de Pterygoplichthys spp.}

En la figura 2 se presentan los valores de las concentraciones totales de los metales pesados analizados en el tejido muscular de Pterygoplichthys spp. en nueve sitios pertenecientes a la cuenca GrijalvaUsumacinta. El Mn y el Cr fueron los metales con mayor presencia en el tejido muscular. Este patrón es consistente con lo observado en la zona, donde esos metales son los de mayor presencia en los sedimentos de la cuenca Grijalva-Usumacinta (SGM 2015).

Teniendo en cuenta todos los sitios muestreados y ambas subcuencas las concentraciones medias de los metales fueron: Mn 734.70 \pm 984.36, Cr 315.16 \pm $192.64, \mathrm{~Pb} 204.62 \pm 141.53$ y Cd $38.04 \pm 8.32 \mu \mathrm{g} / \mathrm{kg}$ (Fig. 2).

Los valores máximos y mínimos de Mn en tejido muscular de Pterygoplichthys spp. fueron 3117.27 y $81.90 \mu \mathrm{g} / \mathrm{kg}$ encontrados en los sitios LSG y BA, la concentración promedio de Mn considerando todos los sitios evaluados fue de $734.70 \pm 984.36 \mu \mathrm{g} / \mathrm{kg}$. Actualmente no se cuenta con estudios referentes en la zona. Sin embargo, Abdel-Khalek (2015) en su estudio de peces en la cuenca del río Nilo encontró valores similares de $\mathrm{Mn}(1000 \pm 240 \mu \mathrm{g} / \mathrm{kg})$ para el pez de granja Oreochromis niloticus, una especie de amplio consumo humano y concentraciones de $2200 \pm$ $600 \mu \mathrm{g} / \mathrm{kg}$ para la misma especie en ambiente natural con descargas industriales cercanas. Ambas especies, Oreochromis niloticus y Pterygoplichthys spp. se alimentan de fauna bentónica, lo que hace que ambos organismos sean susceptibles a bioacumular contaminantes asociados a sedimentos como los metales pesados (Dhanakumar et al. 2015). 

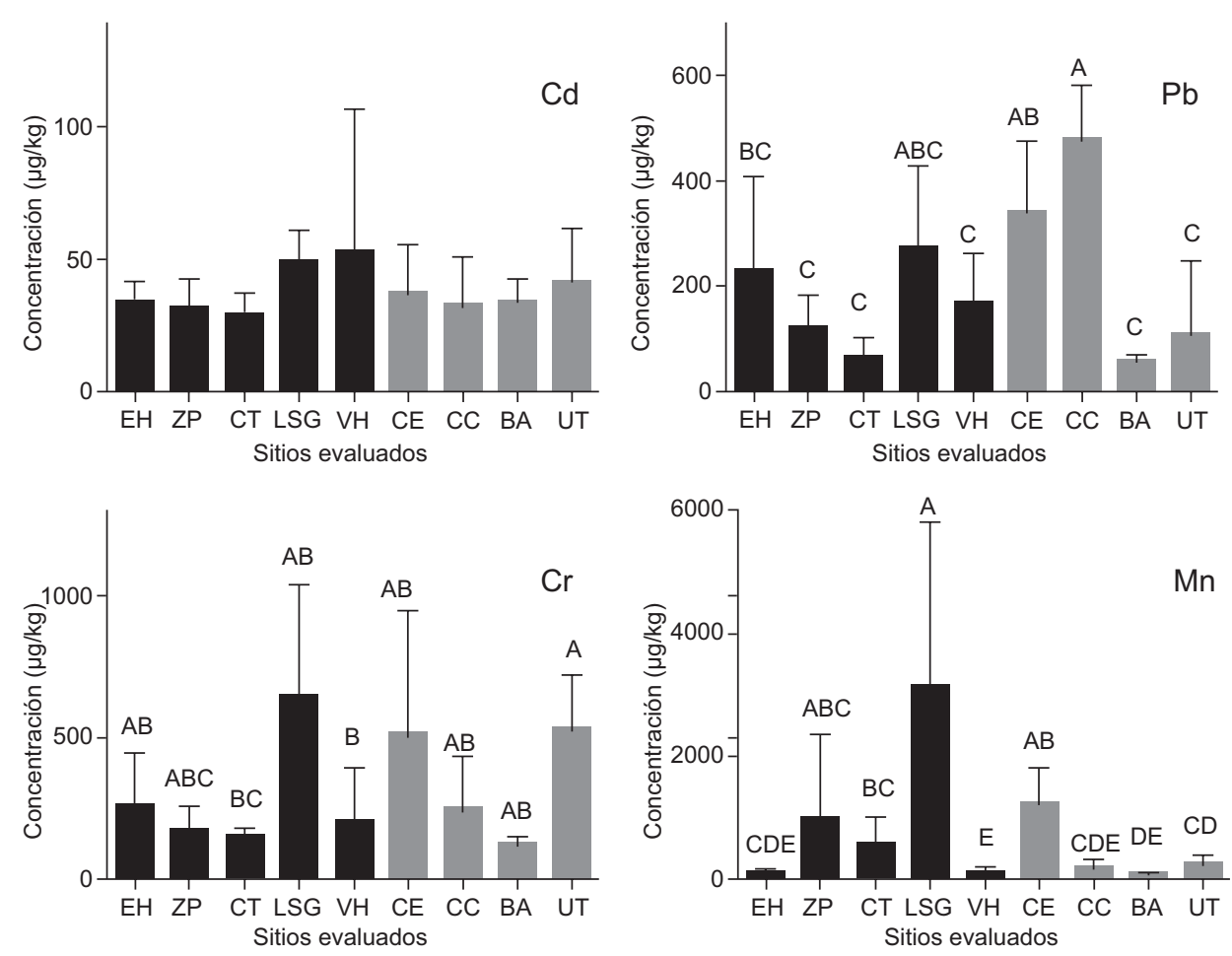

Subcuenca Grijalva

Subcuenca Usumacinta

Fig. 2. Comparación de la concentración promedio de $\mathrm{Cd}, \mathrm{Cr}, \mathrm{Mn}$ y $\mathrm{Pb}$ en tejido muscular de Pterygoplichthys spp. de las subcuencas Grijalva y Usumacinta. Letras diferentes indican diferencias estadísticamente significativas $(\mathrm{p}<0.05)$. Los datos son la media \pm desviación estándar

El Mn es un micronutriente esencial para muchas reacciones bioquímicas en los seres vivos, sin embargo, en concentraciones superiores a su RfD ha sido asociado con problemas en el sistema nervioso central (USEPA 1996b).

Los valores máximos y mínimos detectados para Cr en tejido muscular de Pterygoplichthys spp. fueron $645.15 \mu \mathrm{g} / \mathrm{kg}$ en el sitio LSG y $125.34 \mu \mathrm{g} / \mathrm{kg}$ en BA respectivamente y una concentración media considerando todos los sitios evaluados de $315.16 \pm 192.64 \mu \mathrm{g} / \mathrm{kg}$. Dhanakumar et al. (2015) obtuvieron concentraciones de $1270 \pm 1230 \mu \mathrm{g} / \mathrm{kg}$ en la especie bentopelagica Catla catla, la cual es de amplio consumo humano en la cuenca del río Cauvery de la India que se encuentra influenciado por diversos aportes industriales. Los valores reportados para la India superan por aproximadamente cuatro veces a los encontrados en el presente estudio en Pterygoplichthys spp.

Las concentraciones máximas y mínimas de $\mathrm{Cd}$ en tejido muscular de Pterygoplichthys spp. fueron 53.02 y $28.93 \mu \mathrm{g} / \mathrm{kg}$ correspondientes a los sitios VH y CT respectivamente y una concentración promedio considerando todos los sitios evaluados de $38.04 \pm 8.32 \mu \mathrm{g} / \mathrm{kg}$.
Ernawati (2014) reportó concentraciones de Cd en tejido muscular de Pterygoplichthys pardalis en la cuenca del río Ciliwung, en Indonesia, menores a $5 \mu \mathrm{g} / \mathrm{kg}$. Las concentraciones del presente estudio para Pterygoplichthys spp. en la cuenca del Grijalva-Usumacinta son aproximadamente siete veces mayores a las reportadas por Ernawati (2014).

Los valores máximos y mínimos de $\mathrm{Pb}$ en tejido muscular fueron 481.01 y $56.29 \mu \mathrm{g} / \mathrm{kg}$ para los sitios CC y BA respectivamente y un valor medio considerando todos los sitios evaluados de $204.62 \pm 141.53 \mu \mathrm{g} / \mathrm{kg}$. Ernawati (2014) encontró concentraciones de $2880 \pm 1930 \mu \mathrm{g} / \mathrm{kg}$ en tejido muscular de Pterygoplichthys pardalis en la cuenca del río Ciliwung en Indonesia, lo que es aproximadamente catorce veces mayor a las concentraciones reportadas en el presente estudio. La concentración de $\mathrm{Pb}$ en tejido de P. pardalis reportada por Ernawati se relacionó positivamente con la concentración del metal encontrada en sedimentos, esto atribuible a la bioacumulación y como consecuencia de los aportes industriales cercanos a la cuenca del río Ciliwung. 


\section{Estimación de riesgo de exposición}

La ingesta diaria estimada de metales pesados se comparó con la RfD establecida para el desarrollo de algunos efectos adversos en la salud (Cuadro I). De acuerdo con los métodos estándar de la Agencia de Protección Ambiental (USEPA, por sus siglas en inglés), el riesgo de efecto tóxico se expresa como la relación de la dosis resultante de la exposición a los medios contaminados de un sitio con la dosis de seguridad. Esta relación se llama cociente de riesgo (CR). La probabilidad del desarrollo de los efectos crónicos tóxicos tiende a aumentar con el CR. Las RfD orales fueron $\mathrm{Cd}=1 \times 10^{-3} \mathrm{mg} / \mathrm{kg} /$ día para efecto de proteinuria significativa, $\mathrm{Cr}=1.5 \mathrm{mg} / \mathrm{kg} /$ día para efecto de necrosis celular y Mn $1.4 \times 10^{-1} \mathrm{mg} / \mathrm{kg} /$ día para efecto de daños en el sistema nervioso central (USEPA 1994b, 1996b, 1998b).

En el cuadro I se muestran los parámetros utilizados con los que se calcularon los CR por exposición a Cd, Cr y Mn derivado de la ingesta de Pterygoplichthys spp. obtenidos por el método de simulación probabilística Monte Carlo para las subcuencas Grijalva, Usumacinta y la conjunción de ambas descrita como Grijalva-Usumacinta.

En el cuadro II se resumen las estimaciones del porcentaje de población cuya exposición superaría al menos en un orden de magnitud la dosis de referencia $(\mathrm{CR}>1)$ para las subcuencas Grijalva, Usumacinta y la conjunción de ambas descrita como GrijalvaUsumacinta. En todos los casos, las concentraciones de los elementos analizados fueron mayores en la subcuenca Grijalva, por esta razón, se obtuvo que para el $\mathrm{Cd}$, un $1.63 \%$ de la población estaría expuesta a niveles mayores que la RfD indicada para ese elemento. Mientras que para el Mn, $0.01 \%$ de la población estaría expuesta a niveles superiores a

CUADRO II. ESTIMACIONES DEL PORCENTAJE DE POBLACIÓN CUYA EXPOSICIÓN A Cd, Cr Y Mn SUPERARÍA LAS DOSIS DE REFERENCIA, POR INGESTA DE TEJIDO MUSCULAR DE Pterygoplichthys spp. EN LAS SUBCUENCAS GRIJALVA Y USUMACINTA ASÍ COMO LA CONJUNCIÓN DE AMBAS

\begin{tabular}{lccc}
\hline \multicolumn{3}{c}{ Cociente de Riesgo } \\
\hline Metal & $\begin{array}{c}\%>1 \\
\text { Subcuenca } \\
\text { Grijalva }\end{array}$ & $\begin{array}{c}\text { Subcuenca } \\
\text { Usumacinta }\end{array}$ & $\begin{array}{c}\%>1 \\
\text { Grijalva- } \\
\text { Usumacinta }\end{array}$ \\
\hline $\mathrm{Cd}$ & 1.63 & 0.00 & 0.81 \\
$\mathrm{Cr}$ & 0.00 & 0.00 & 0.00 \\
$\mathrm{Mn}$ & 0.01 & 0.00 & 0.00 \\
\hline
\end{tabular}

los límites de seguridad y finalmente para el Cr, la probabilidad de que la población supere los límites de la exposición a este metal derivada de la ingesta de Pterygoplichthys spp. es prácticamente nula.

Actualmente las autoridades en materia pesquera y los gobiernos locales en los sitios donde se realizó el presente estudio han promovido el consumo de carne de este pescado debido a muchos beneficios nutricionales. Esto se debe principalmente al contenido de proteína de alta calidad, así como a un alto contenido de dos tipos de ácidos grasos omega-3 polisaturados: ácido eicosapentaenoico y ácido docosahexaenoico presentes en diferentes especies de peces comestibles en comparación con otros productos de origen animal (Castro-González 2002, Clarkson 2002, Domingo et al. 2007) y con beneficios en la salud para la población (Din et al. 2004, Ismail 2005). Sin embargo, el contenido de metales tóxicos en carne de pescado puede contrarrestar los efectos positivos de su consumo. Chan y Egeland (2004), Has-Schön et al. (2006) y Castro y Méndez (2008) observan concentraciones de metales en peces de agua dulce por lo que esta condición debe ser suficientemente valorada antes de proponer una política pública que establezca su consumo frecuente en estos escenarios.

A pesar de que los riesgos de exposición a los metales contenidos en los peces de ambas cuencas fueron bajos, existen reportes que indican que algunos procesos culinarios alteran las propiedades de los contaminantes, produciendo un efecto de aumento de concentración atribuido a la pérdida de agua (Ruelas et al. 2011, Kalogeropoulus et al. 2012, Costa et al. 2013).

\section{Estimación de la exposición a Pb por IEUBK}

El cuadro III resume la estimación del porcentaje de niños de 36 a 60 meses de edad con probabilidad de tener niveles de $\mathrm{Pb}$ en sangre superiores a $5 \mathrm{y}$ $10 \mu \mathrm{g} / \mathrm{dL}$ derivados de la ingesta de Pterygoplichthys spp. Estos valores son aquellos a que el Centro de Control de Enfermedades de EU (CDC, por sus siglas en inglés) ha establecido como de referencia por haber asociado efectos adversos en poblaciones con esos niveles (USEPA 1998b, ATSDR 2007).

Para la subcuenca Grijalva se estima que un $2.79 \%$ de la población infantil tendría concentraciones superiores a $5 \mu \mathrm{g} \mathrm{Pb} / \mathrm{dL}$ en sangre, mientras que para la subcuenca Usumacinta $7.24 \%$ de los infantes presentaría concentraciones que sobrepasen este mismo punto de corte. De la misma forma, en la subcuenca Grijalva la población infantil con concentraciones superiores a $10 \mu \mathrm{g} \mathrm{Pb} / \mathrm{dL}$ de sangre sería de $0.035 \%$ y para la subcuenca Usumacinta de $0.168 \%$. 
CUADRO III. ESTIMACIÓN DE LA PROPORCIÓN DE POBLACIÓN CON CONCENTRACIONES SANGUÍNEAS DE PLOMO A NIVELES SUPERIORES AL DESARROLLO DE EFECTOS ADVERSOS EN LAS SUBCUENCAS GRIJALVA Y USUMACINTAASÍ COMO LA CONJUNCIÓN DE AMBAS

\begin{tabular}{lccc}
\hline \multicolumn{4}{c}{ IEUBK } \\
\hline Sitio & $\begin{array}{c}\text { Edad } \\
\text { (meses) }\end{array}$ & $\begin{array}{c}\%>5 \\
(\mu \mathrm{g} \mathrm{Pb} / \mathrm{dL} \\
\text { sangre })\end{array}$ & $\begin{array}{c}\%>10 \\
(\mu \mathrm{g} \mathrm{Pb} / \mathrm{dL} \\
\text { sangre })\end{array}$ \\
\hline $\begin{array}{l}\text { Subcuenca Grijalva } \\
\text { Subcuenca Usumacinta }\end{array}$ & $36-60$ & 2.792 & 0.035 \\
Grijalva-Usumacinta & $36-60$ & 7.254 & 0.168 \\
\hline
\end{tabular}

Para el resto de la población, a pesar de que las estimaciones de las concentraciones sanguíneas parecen ser bajas, se debe tener especial cuidado puesto que los niños son los más vulnerables a la exposición $\mathrm{a} \mathrm{Pb}$ debido a que el sistema nervioso es el órgano blanco principal y no se ha establecido aún un nivel considerado como aceptable (ATSDR 2007).

\section{CONCLUSIONES}

El presente trabajo se centra en el análisis de un escenario de consumo infantil generalizado del pez invasor Pterygoplichthys spp. con diferentes cargas corporales de metales pesados en tejido muscular. Esta consideración involucra la ingesta frecuente por parte de las comunidades ribereñas rurales que han sido afectadas por el desmesurado incremento de la población de este género. La estimación de los riesgos a la salud en las comunidades ribereñas del río Grijalva y el río Usumacinta por la exposición a $\mathrm{Cd}, \mathrm{Cr}, \mathrm{Mn}$ y $\mathrm{Pb}$ contenidos en tejido muscular de Pterygoplichthys spp. se realizó bajo el procedimiento descrito por Díaz-Barriga (1999).

En términos generales, las concentraciones encontradas de $\mathrm{Cd}$, Cr y Mn en este estudio fueron bajas (Ernawati 2014, Frías et al. 2014, Abdel-Khalek 2015, Dhanakumar et al. 2015). Sin embargo, se refleja la condición de vulnerabilidad de los escenarios estudiados, donde si bien no hay minería ampliamente desarrollada, las concentraciones ambientales de los metales analizados son suficientes para integrarse a las redes tróficas y generar una exposición potencialmente nociva para las poblaciones humanas.

En el presente trabajo se encontraron concentraciones mayores de $\mathrm{Mn}$ y Cr en tejidos musculares del pez respecto a los otros metales cuantificados $(\mathrm{Pb}$ y $\mathrm{Cd})$, pero éstas no representaron un riesgo alto para generar un efecto adverso por su ingesta crónica. Sin embargo, en un escenario real existe una exposición conjunta a varios contaminantes y esta condición no se ha valorado aún.

Así, se requiere evaluar otras fuentes de exposición $\mathrm{a} \mathrm{Pb}$ que podrían alterar las concentraciones en sangre infantil, como son el esmalte utilizado en la fabricación de barro vidriado que se emplea tradicionalmente en México para cocinar, almacenar y servir alimentos.

Es necesario realizar un análisis más detallado involucrando otros factores como la talla de los peces, el estadio biológico, la diferenciación entre sexos, la diferenciación entre tejidos, así como evaluar si existen diferencias dependiendo de la estación del año o la presencia otros contaminantes inorgánicos como el arsenico y el mercurio y orgánicos como los plaguicidas.

De forma paralela hay que realizar una valoración bromatológica que refleje los aportes nutricionales asociados a la ingesta de Pterygoplichthys spp. para establecer un mejor balance del riesgo-beneficio del consumo de este pez.

Por otra parte hace falta evaluar si los procesos de cocina o transformación de la materia prima al desarrollar subproductos con Pterygoplichthys spp. propician un efecto de aumento en la concentración de los contaminantes haciéndolos inseguros para su integración periódica en la dieta humana.

Este estudio se interpreta como una valoración de la exposición potencial a metales pesados por consumo habitual de Pterygoplichtys. Es además útil para determinar las zonas donde su consumo es seguro y finalmente busca la incorporación de criterios de salud pública y salud ambiental dentro de las estrategias sugeridas para abatir el problema generado por la invasión biológica en estos escenarios.

\section{AGRADECIMIENTOS}

Este trabajo fue apoyado por el Consejo Nacional de Ciencia y Tecnología (CONACyT) convenio 290747 No. 355435 . CONACyT Proyecto CB-200801-106050. PRODEP: DSA/103.5/14/10379. ID Sol: 155718 .

\section{REFERENCIAS}

Abdel-Khalek A. A. (2015). Risk assessment, bioaccumulation of metals and histopathological alterations in Nile tilapia (Oreochromis niloticus) Facing degraded aquatic conditions. Bull. Environ. Contam. Toxicol. 94, 77-83. DOI: $10.1007 / \mathrm{s} 00128-014-1400-9$. 
ATSDR (2007). Resumen de salud publica: Plomo. Agencia para Sustancias Tóxicas y el Registro de Enfermedades. Resumen. Atlanta GA, EUA, 15 pp.

Barba-Macías E., Juárez-Flores J. y Magaña-Vázquez M. (2014). Nuevos registros de plecos (Pterygoplichthys pardalis) (Siluriformes: Loricariidae) en las cuencas del río Grijalva y Tonalá, Pajonal-Machona, Tabasco. En: Especies invasoras acuáticas. Casos de estudio en ecosistemas de México. (A. M. Low Pfeng, P. A. Quijón, E. M. Peters Recagno, Eds.) Secretaría de Medio Ambiente y Recursos Naturales, Ciudad de México, México, 658 pp.

Bervotes L., Blust R. y Verheyen R. (2001). Accumulation of metals in the tissue of three spined sticklebacks (Gasterosteus aculeatus) from natural fresh waters. Ecotoxicol. Environ. Saf. 48, 117-127. DOI: 10.1006/ eesa.2000.2010.

Burger J., Gaines K. F., Boring S., Syephans L., Snodgrass J. y Dixon C. (2002). Metals levels in fish from the Savannah River: potential hazards to fish and other receptors. Environ. Res. 89, 95-97. DOI: 10.1006/ enrs.2002.43.

Cano Salgado M. P., Bello Baltazar E. y Barba, E. (2012). Innovación social y capacidad de organización de las cooperativas pesqueras en el municipio de Balancán, Tabasco, México. Estudios Sociales 20, 65-98.

Castro Díez P., Valladares F. y Alonso A. (2004). La creciente amenaza de las invasiones biológicas. Ecosistemas 13, 61-68.

Castro M. I. y Méndez M. (2008). Heavy metals: Implications associated to fish consumption. Environ. Toxicol. Pharmacol. 26, 263-271. DOI:10.1016/j. etap.2008.06.001.

Castro-González I. M. (2002). Ácidos grasos omega-3: beneficios y fuentes. Interciencia 27, 128-136.

Chan M. H. y Egeland M. G. (2004). Fish consumption, mercury exposure, and heart disease. Nutr. Rev. 62, 6872. DOI: http://dx.doi.org/10.1111/j.1753-4887.2004. tb00027.x.

Chávez J. M., de la Paz R. M., Manohar S. K., Pagulayan R. C. y Carandang J. R. (2006). New Philippine record of South American sailfin catfishes (Pisces: Locariidae). Zootaxa 1109, 57-68.

Clarkson W. T. (2002). The three modern faces of mercury. Environ. Health Perspect. 110, 11-23.

Collares-Pereira M. J., Cowx J. I., Rodríguez J. A. y Rogado L. (2002). A conservation strategy for Anaecypris hispanica: a picture of life for a highly endangered Iberian fish. En: Conservation of freshwater fishes: Options for the future. (M. J. M. Collares- Pereira, M. Coelho e I. Cowx, Eds.). Blackwell Science, Oxford, Algarve, Portugal, 462 pp.

Costa S., Afonso C., Bandarra N. M., Gueifão., Castanheira I., Carvalho M. L., Cardoso C. y Nunes M. L.
(2013). The emerging farmed fish species meagre (Argyrosomus regius): How culinary treatment affects nutrients and contaminants concentration and associated benefit-risk balance. Food and Chem. Toxicol. 60, 277-285. DOI: 10.1016/j.fct.2013.07.050.

Dhanakumar S., Solaraj G. y Mohanraj R. (2015). Heavy metal partitioning in sediments and bioaccumulation in commercial fish species of three major reservoirs of river Cauvery delta region, India. Ecotoxicol. Environ. Saf. 113, 145-151. DOI: 10.1016/j.ecoenv.2014.11.032.

Díaz-Barriga F. (1999). Metodología de identificación y evaluación de riesgos para la salud en sitios contaminados. 1a ed. Organización Panamericana de la Salud. Centro Panamericano de Ingeniería Sanitaria y Ciencias del Ambiente. Lima, Perú, 42 pp.

Din J. N., Newby D. E. y Flapan A. D. (2004). Omega 3 fatty acids and cardiovascular disease-fishing for natural treatment. BMJ. 328, 30-35. DOI: 10.1136/ bmj.328.7430.30.

Domingo J. L., Bocio A., Flaco G. y Llobet J.M. (2007). Benefits and risks of fish consumption. Part I. A quantitative analysis of the intake of omega-3 fatty acids and chemical contaminants. Toxicology. 230, 219-226. DOI: 10.1016/j.tox.2006.11.054.

Dukes J. S. y Mooney H. A. (1999). Does global change increase the success of biological invaders? TREE. 14, 135-139. DOI: 10.1016/S0169-5347(98)01554-7.

Ernawati Y. (2014). The analysis of the concentration of heavy metals cadmium, mercury and lead in the flesh of suckermouth catfish (Pterygoplichthys pardalis) in Ciliwung River, Indonesia. AACL Bioflux. 7, 33- 42.

Escalera-Gallardo C., Arroyo-Damián M., Moncayo-Estrada R. y Zarazúa J. A. (2012). Pesquería sustentable y desarrollo local. Uso y aprovechamiento potencial del Pez Diablo. En: Desarrollo local y empresa (M. Cárcamo Solis, M. Martín Carbajal, A. Ortega Hernández, Eds.). Universidad de Guanajuato, Guanajuato, México, $281 \mathrm{pp}$.

Ferraris C. J. (2007). Checklist of catfishes, recent and fossil (Osteichthyes: Siluriformes), and catalogue of siluriform primary types. Zootaxa 1418, 300 .

Flores J. y Albert L. A. (2004). Environmental lead in Mexico 1990-2002. Rev. Environ. Contam. Toxicol. 181, 37-109. DOI: 10.1007/0-387-21733-9_2.

Frías Espericueta M. G., Osuna López J. I., Izaguirre Fierro G., Aguilar Juárez M. y Voltolina D. (2010). Cadmio y Plomo en organismos de importancia comercial de la zona costera de Sinaloa, México: 20 años de estudios. CICIMAR Oceánides. 25, 27-39.

Frías M. G., Zamora F. K., Osuna J. I., Muy M. D., Rubio W., Aguilar M. y Voltolina D. (2014). Cadmium, cooper, lead, and zinc contents of fish marketed in NW 
Mexico. Scientific World Journal 2014,1-4. DOI: http:// dx.doi.org/10.1155/2014/546897.

Guzmán A. F. y Barragán S. J. (1997). Presencia de bagres sudamericanos (Osteichthyes: Loricariidae) en el río Mezcala, Guerrero, México. Vertebrata Mexicana 3, 1-4.

Has-Schön E., Bogut I. y Strelec I. (2006). Heavy metal profile in five fish species included in human diet, domiciled in the end flow of River Neretva (Croatia). Arch. Environ. Contam. Toxicol. 50, 545-551. DOI: 10.1007/s00244-005-0047-2.

Hoover J. J., Killgore K. J. y Cofrancesco A. F. (2004). Suckermouth catfishes: threats to aquatic ecosystems of the United States? Aquatic Nuisance Species Research Program. Engineers Research and Development Center, Vicksburg, MS. ANSRP Bulletin 4, 1-13.

Hossain M. Y., Rahman M. M., Ahmed Z. F., Ohtomi J. y Islam A. (2008). First record of the South American sailfin catfish Pterygoplichthys multiradiatus in Bangladesh. J. Appl. Ichthyol. 24, 718-720. DOI: 10.1111/j.1439-0426.2008.01117.x.

Ismail H. M. (2005). The role of omega-3 fatty acids in cardiac protection: on overview. Front. Biosci. 10, 1079-1088. DOI: http://dx.doi.org/10.2741/1601.

Kalogeropoulos N., Karavoltsos S., Sakellari A., Avramidou S., Dassenakis M. y Scoullos M. (2012). Heavy metals in raw, fried and grilled Mediterranean finfish and shellfish. Food and Chemical Toxicology 50, 37023708. DOI:10.1016/j.fct.2012.07.012.

Keane R. M. y Crawley M. J. (2001). Exotic plant invasions and the enemy release hypothesis. TREE. 17, 164-170. DOI: 10.1016/S0169-5347(02)02499-0.

Mendoza R., Contreras S., Ramírez C., Koleff P., Álvarez P. y Aguilar V. (2007). Los peces diablo: especies invasoras de alto impacto. Biodiversitas 70, 1-5.

Mooney H. A. y Hobbs R. J. (2000). Invasive species in a changing world. Island Press, Washington D.C., EUA, 457 pp.

Nelson J. S. (2006). Fishes of the world. 4a ed. John Wiley and Sons Inc., Edmonton, Alberta, Canadá, 424 pp.

Newarman M. C. (1998). Fundamentals of ecotoxicology. Ann Arbor Press, Chelsea, EUA, 278 pp.

Nico L. G., Jelks H. L. y Tuten T. (2009). Non-native suckermouth armored catfishes in Florida: Description of nest burrows and burrow colonies with assessment of shoreline conditions. ANSRP Bulletin 9, 1-30.

Nico L. G. y Martin R. T. (2001). The South American suckermouth armored catfish, Pterygoplichthys anisitsi (Pisces: Loricariidae) in Texas, with comments on foreign fish introductions in the American Southwest. South. Nat. 46, 98-104. DOI: 10.2307/3672381.

Paramo D., Hernández S. M. y Perera G. (2008). Alternativas de aprovechamiento de Pterygoplichthys pardalis, mejor conocido como "pez diablo" o "plecos" en Tabasco. En: Manejo, procesamiento primario y transformación de los productos pesqueros con énfasis en los recursos de la Reserva de la Biosfera Pantanos de Centla. (F. Arévalo, L. Hernández, M. Mendoza-Carranza, Eds.). El Colegio de la Frontera Sur, Villahermosa, Tabasco, México. 95 pp.

Ramírez-Guevara N. N. y Rodiles-Hernández R. (2013). El impacto de especies invasoras: El caso de la familia Loricariidae (Siluriformes). En: La biodiversidad en Chiapas: Estudio de Estado Volumen II. (A. Cruz, E. D. Melgarejo, F. Camacho, K. C. Nájera, Eds.). Comisión Nacional para el Conocimiento y Uso de la Biodiversidad (CONABIO) y Gobierno del Estado de Chiapas, México, $431 \mathrm{pp}$.

Ruelas J., Páez F., Ruiz A. C. y Zamora N. (2011). Health risk associated to dietary intake of mercury in selected coastal areas of Mexico. Bull. Environ. Toxicol. 86, 180188. DOI: 10.1007/s00128-011-0189-z.

Santamaría L., Pericás J., Carrete M. y Tella J. L. (2008). La ausencia de enemigos naturales favorece las invasiones biológicas. En: Invasiones biológicas. (M. Vilà, F. Valladares, A. Traveset, L. Santamaría, P. Castro, Eds.). Consejo Superior de Investigaciones Científicas (CSIC). Madrid, España, 215 pp.

SGM (2015). GeoInfoMex. Servicio Geológico Mexicano. [en línea]. http://goo.gl/IQXqNN 02/10/2015.

USEPA (1994a). \#9285.7-21. Validation strategy for the integrated exposure uptake biokinetic model for lead in children. United States Environmental Protection Agency. Manual. Washington, D.C., EUA, 23 pp.

USEPA (1994b). \#7440-43-9 Cadmium. Integrated risk information system. [en línea] http://goo.gl/Bruys1 02/10/2015.

USEPA (1996a). METHOD 3052 [en línea] http://goo.gl/ WCsaoh 02/10/2015.

USEPA (1996b). \#7439-96-5 Manganese. Integrated risk information. [en línea] http://goo.gl/eUzcpQ 02/10/2015.

USEPA(1997). EPA/630/R-97/001 Guiding principles for Monte Carlo analysis. U.S. Environmental Protection Agency. Manual. Washington, D.C., EUA, 35 pp.

USEPA (1998a). \#9200.4-27P Memorandum: oswer directive: Clarification to the 1994 revised interim soil lead $(\mathrm{Pb})$ guidance for CERCLA sites and RCRA corrective action facilities. United States Environmental Protection Agency. Memorandum. Washington, D.C., EUA, 14 pp.

USEPA (1998b). \#16065-83-1 Chromium (III), insoluble salts. Integrated risk information system. [en línea] http://goo.gl/vvAOcA 02/10/2015.

USEPA (2007a). METHOD 7010 [en línea] http://goo.gl/ de5Vry 02/10/2015. 
USEPA (2007b). \#9285.7-42 User's guide for the integrated exposure uptake biokinetic model for lead in children (IEUBK) Windows ${ }^{\circledR}$. Syracuse Research Corporation. Manual. Running Ridge Road North Syracuse, N.Y., EUA, 46 pp.

USEPA (2008). EPA/600/R-06/096F Child-Specific exposure factors handbook. United States Environmental Protection Agency. Reporte. Washington, D.C., EUA, 687 pp.

Villanueva S. F. y Botello A. V. (1998). Metal polution coastal areas of Mexico. Rev. Contam. Toxicol. 157, 53-94. DOI: 10.1007/978-1-4612-0625-5 3.

Wakida-Kusunoki A. T. y Amador del Ángel E. L. (2011). Aspectos biológicos del plecos invasor Pterygoplichthys pardalis (Teleostei: Loricariidae) en el río Palizada, Campeche, México. Rev. Mex. Biodiv. 82, 870-878. DOI: http://dx.doi.org/10.7550/rmb.27842.
Wakida-Kusunoki A. T., Ruiz-Carus R. y Amador del Ángel E. L. (2007). Amazon sailfincatfish, Pterygoplichthys pardalis (Casternau, 1855) (Loricariidae), another exotic species established in southeastern Mexico. The Southwestern Naturalist 52, 141-144. DOI:10.1894/0038-4909(2007)52[141:ASCPPC]2. $0 . \mathrm{CO} ; 2$.

Zayas Mujica R. y Cabrera Cárdenas U. (2007). Los tóxicos ambientales y su impacto en la salud de los niños. Rev. Cubana Pediatr. 79, 1-4. 